\title{
Hole Detection and Healing in Hybrid Wireless Sensor Network
}

\author{
Samidha N. Kalwaghe*, Atul V. Dusane \\ Department of Computer Science and Engineering, SSBT COET Jalgaon, India \\ *Corresponding author, email: Atulp_1987@yahoo.co.in
}

\begin{abstract}
The emerging technology of wireless sensor network (WSN) is expected to provide a broad range of applications, such as battlefield surveillance, environmental monitoring, smart spaces and so on. The coverage problem is a fundamental issue in WSN, which may cause due to low residual energy of nodes or poor installment. But in order to get full coverage of sensing area Coverage problem must be avoided. If the problem is unavoidable the coverage hole must be healed. Current hole healing algorithms uses complicated hole detection strategies like TENT rule. This project seeks to address the problem of hole detection and healing in mobile WSNs by deploying mobile sensors in the network, which is called hybrid sensor network. An enhanced hole detection and healing method (MHEAL) is proposed. MHEAL is a distributed and localized algorithm that operates in two distinct phases. First, is Distributed Hole Detection (DHD) proposed to identify the boundary nodes and discover holes. Second, is hole healing which uses a virtual forces based hole healing approach where only the nodes located at an appropriate distance from the hole and the nodes having maximum energy will be involved in the healing process. Unlike existing algorithms, proposed algorithm uses QURD based node detection and energy efficient Hole healing and thus solves the problem of hole with $100 \%$ coverage, minimum node movements and minimum node distance travelled thus giving a cost efficient solution.
\end{abstract}

Keywords: TENT Rule, MHEAL, HEAL, DHD, QUARD Rule, Hybrid sensor network

Copyright (C) 2016 APTIKOM - All rights reserved.

\section{Introduction}

Wireless Sensor Network (WSN) is formed from the tiny sensors. Today Wireless sensor network is used in every field of applications like battle field, traffic control, in colleges, entry gates etc. An area where a group of sensor nodes stops working and does not take part in data sensing and communication is termed as a hole in the network. Holes are the barriers for communication with huge impact on the performance of the network. Hole detection identifies damaged, attacked or inaccessible nodes. Struck nodes are defined where packets can get struck doing routing. WSN needs the regular monitoring of network because of continuous change is possible as the sensors may fail. Target Coverage problem is concerned with the random deployment of sensor nodes for monitoring the specific targets for maximum duration. It is crucial to maintain the connectivity among sensors so that they can communicate with each other and with the base-station. Most of the existing solutions use global operations to calculate the size of a big hole and then relocate a group of mobile sensors to heal the hole. Some existing localized solutions requires strong assumptions or even unrealistic ones. The problem is to design a mechanism for detecting and recovering holes by exploiting only the nodes mobility. Exploiting the nodes mobility is not possible in static WSN and hence nodes need to be deployed in hybrid WSN. Healing of holes needs displacement of nodes which may require huge movement of nodes, leading to energy depletion of sensor network. Current hole detection process use TENT Rule for finding the struck nodes [2]. Many related works based on hole identification and border detection has been done. Summarization of work done by some researchers in coverage hole detection and healing algorithm is done here W. Chu and K. Ssu [1], proposed a Decentralized Boundary Detection (DBD) algorithm to identify sensor nodes near a hole or obstacle in WSN using topological approach. Each node knows its three-hop neighbors by exchanging HELLO messages, one-hop, and two-hop node information. Each node then constructs 2-hop neighbor graph. If cycle exists in such a graph then there is a hole in network. For dealing with hole which is not 
included totally inside 2-hop neighbor graph, another rule based on contour structure was developed. Detection of broken contour line implies either network boundary or an obstacle.

Z. J. Zhang et.al.,2013 used Delaunay triangulation to detect the coverage holes. The set of static nodes are triangulated into many triangles and the circumcenter of every triangle is calculated. Then this algorithm detects whether the circumcenter is covered by the three static sensors that formed the triangle. If the circumcenter is covered, it is not a coverage hole. Most of the above methods use the disc sensing model since it will simplify analysis. However, this model is not realisticI.M. Khan et al., 2012 Hopbased approach for holes and boundary detection in wireless sensor networks [3] proposed a hop based approach to find holes in sensor networks. There are three phases namely, information collection phase where each node exchanges information to build a list of $\mathrm{x}$-hop neighbors, path construction phase where communication links between sensor nodes in list of $\mathrm{x}$-hop neighbors are identified and finally path checking phase where paths are examined to infer boundary and inner nodes. If the communication path of x-hop neighbors of a node is broken then it is boundary node. Algorithm works for node degree of 7 or higher which is better than some of the other approaches but there is a huge communication overhead involved to identify $\mathrm{x}$-hop neighbors.

\section{Research Method}

A modified hole detection and healing method (MHEAL) is proposed, that could remove the drawbacks of existing algorithms. Proposed method is a distributed and localized algorithm that operates in two distinct phases. First, is Distributed Hole Detection (DHD) proposed to identify the boundary nodes and discover holes. Second, is hole healing which uses a virtual forces based hole healing algorithm. Unlike existing algorithms, proposed algorithm uses QURD based node detection method and could be cost efficient as node selection depends upon the lowest residual energy node.

\subsection{Hole Detection}

\subsubsection{Struck node Identification}

Presence of hole in a region is identified by the presence of struck which could be detected using QUARD Rule. Each node divides the region into 4 quadrants by keeping itself as center and checks for neighbors in each region. The QUARD Rule states that If a node is not having at least single neighbor in any of the quadrant it is considered as stuck node.

\subsubsection{Border Detection}

The Struck nodes also contains the border nodes, so we need to separate those border nodes from the struck nodes Xmax, Ymax, Xmin, Ymin. will give the complete position of boundary nodes nodes having this values are the boundary nodes

\subsubsection{Hole Discovery}

It selects two nodes $b_{n}$ and $b_{m}$ among the struck nodes so that the distance between them is the longest between any two nodes in the set of boundary nodes

Distance $(b m, b n)=\operatorname{Max}\left\{\right.$ Distance $\left.\left(b_{j}, b_{k}\right) / b_{j}, b_{k} \in\left\{b_{0}, b_{1}, \ldots \ldots, b_{N}\right\}\right\}$

Then, it calculates the hole center, which is the mid-point $v$ of segment $b_{m} b_{n}$

$$
\left.\begin{array}{l}
\mathrm{x}_{\mathrm{v}}=\left(\mathrm{x}_{\mathrm{bm}}+\mathrm{x}_{\mathrm{bn}}\right) / 2 \\
\mathrm{y}_{\mathrm{v}}=\left(\mathrm{y}_{\mathrm{bm}}+\mathrm{y}_{\mathrm{bn}}\right) / 2
\end{array}\right\}
$$

\subsection{Hole Healing}

\subsubsection{HHA Determination:}

To determine the HHA, the radius of the circle that defines the HHA is needed to be defined. To find the appropriate radius, an iterative approach based on the following formula is used

$$
\mathrm{R}=\mathrm{r} *(1+\beta), \beta \in \mathrm{R}^{+}
$$

$\mathrm{r}$ is the hole radius. $\beta$ is a positive constant, which depends on the nodes density and the sensing range Rs. For $\beta=0$, we start with a radius equals to the hole radius

APTIKOM J. CSIT Vol. 1, No. 2, 2016 : 41-45 
ted hole radius $r$ due to the fact that this area may contain a sufficient nodes to heal the hole. The area

$$
\frac{\pi \mathrm{r}^{2}}{\pi \mathrm{R}_{\mathrm{S}}^{2}}=\frac{\mathrm{r}^{2}}{\mathrm{R}_{\mathrm{S}}^{2}}
$$

defined by this circle (denoted HHA-0) is equal to $\pi r^{2}$. The number of nodes necessary to cover the area HHA-0 is equal to:

If the number of nodes is less than the required number to heal the hole, the movement of these nodes will create new holes. To avoid this scenario a new round is started by increasing ' $\beta$ ', it repeat process until it finds a sufficient number of nodes to recover the hole.

\subsubsection{Healing}

After determining the HHA the nodes involved in the healing process receive Attractive and repulsive forces from the hole-center and this nodes move towards it.

If $\mathrm{Nh}$ is the number of nodes present in the Hole Healing Area, Ns is the number of struck nodes and Number of nodes in HHA other than Struck nodes Nh-s

Than

$\mathrm{Nh}-\mathrm{s}=\mathrm{Nh}-\mathrm{Ns}$

In order to attain maximum coverage, attractive and repulsive forces will be applied on all these nodes. The nodes present in the HHA other than struck nodes will move $2.5 \mathrm{~m}$ due to attractive forces. For energy effect hole healing only fixed nodes having maximum energy will be moved towards the centre. Other nodes position will remain unchanged

If $\mathrm{N}$ is the no of nodes required for healing the hole than every Quadrant must move at least $\mathrm{N} / 4$ nodes Nodes among the overlapping nodes are sorted depending upon their energy levels.3nodes having height energy are considered for hole healing. Thus the complete hole is healed with minimum cost and maximum coverage. The complete architecture of MHEAL is given in Figure 1

Tables and Figures are presented center, as shown below and cited in the manuscript.

Table 1. The Performance of ...

\begin{tabular}{ccc}
\hline Variable & Speed (rpm) & Power $(\mathrm{kW})$ \\
\hline $\mathrm{x}$ & 10 & 8.6 \\
$\mathrm{y}$ & 15 & 12.4 \\
$\mathrm{z}$ & 20 & 15.3 \\
\hline
\end{tabular}

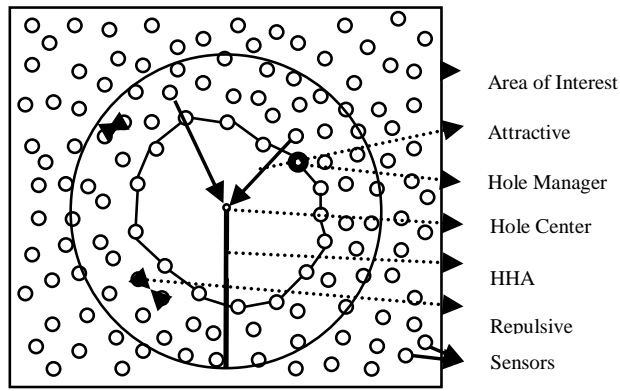

Figure 1. Architecture of MHEAL algorithm

\section{Results and Analysis}

For both HEAL and MHEAL the performance differentials are analyzed using number of movements, total distance travelled and coverage percentage. In 3.13 .2 and 3.3 The result analysis is shown by graphs, The different parameters are consider at y-axis like number of movements, total distance travelled and coverage percentage where number of nodes are kept constant at $\mathrm{x}$-axis. In graph 3.1 it can be seen that when 200 nodes are deployed MHEAL makes total 125 Movements while MHEAL does only 47 Movements Similarly for 250 nodes HEAL movements are 161 while the Movements of MHEAL remains 47 and thus increasing the number of nodes will increase the number of nodes Movement in case of HEAL while MHEAL keeps the number of Movement same and less. In graph 3.2 it can be seen that when 200 nodes are deployed the total distance travelled by hole healing nodes in MHEAL is $391 \mathrm{~m}$ while nodes in IHEAL moves only $258 \mathrm{~m}$ distance. When the number of nodes deployed increases from 200 nodes to 250 nodes than total distance travelled By HEAL increases but 
total distance travelled By MHEAL is found to be decreased .For more than 300 deployed nodes the total distance travelled by HEAL keeps on increases while that of MHEAL decreases. Thus the movement of nodes done in case of MHEAL is less as compared with HEAL.

In graph 3.3 when the number of deployed nodes increases from 200 to 400 both HEAL and MHEAL gives same $100 \%$ coverage i.e. after Executing both the hole detection and healing algorithm all the ROI is sensed correctly. Thus both HEAL and IHEAL gives same result for area coverage.

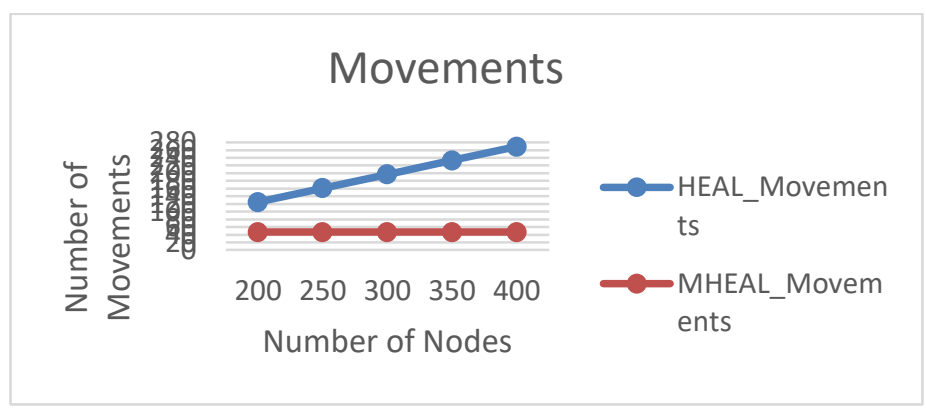

Figure 3.1. Number of Nodes V/S Number of Movements

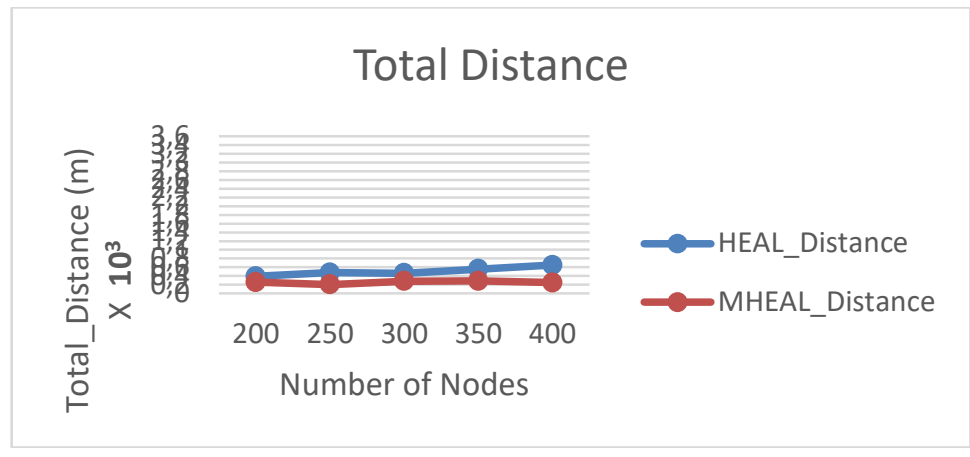

Figure 3.2. Number of Nodes V/S Total Distance

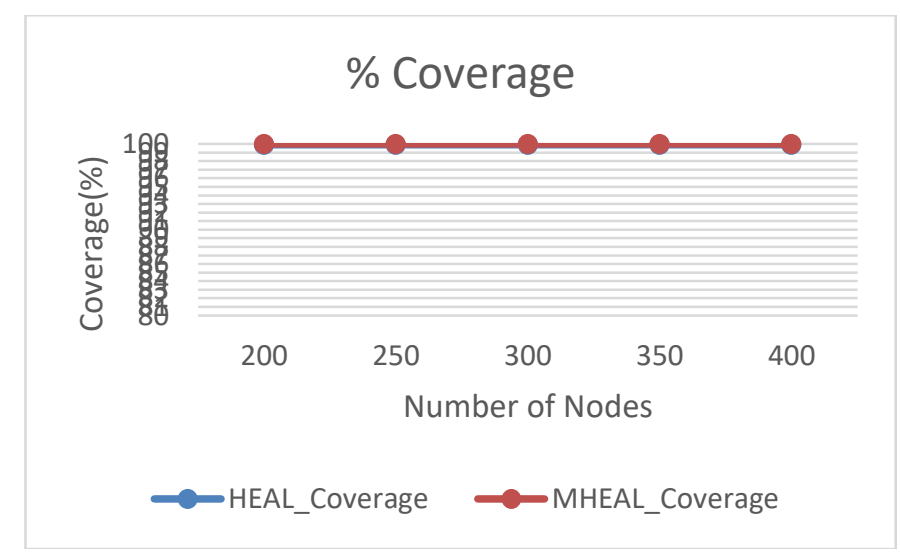

Figure 3.3. Number of Nodes V/S \% Coverag

\subsection{Discussion}

A system is designed to solve the problem of hole detection and healing in hybrid WSN Further enhancements in this system is done to increase its performance. The performance of both the algorithms 
is done by looking at the graphs. It is found that in HEAL the total number of nodes required are just calculated to compute the HHA. This total number of nodes would also have been used to move fixed number of nodes for healing but this is not done in this case and hence as all the nodes in the HHA moves due to attractive and repulsive forces the total number of node movement and total distance travelled by nodes increases, also there is no concern of energy here and hence this strategy may lead to high cost. While in case of MHEAL total number of nodes required are calculated to compute the HHA and this many number of nodes only move in the healing process also here only those nodes are selected to healing which have high residual energy and whose distance from the hole center is less. Due to this the total number of node movement and total distance travelled by nodes is less in hybrid WSN as compared with HEAL and thus MHEAL provides an energy efficient and cost efficient solution for hole detection and healing.

\section{Conclusion}

This project has proposed and implemented a lightweight and comprehensive two-phase protocol, MHEAL, for ensuring area coverage employing a Hybrid mobile WSN. The protocol uses a distributed hole-detection algorithm (DHD) to detect holes in the network. By exploiting the virtual forces concept, this approach relocates only the adequate nodes within the shortest distance and at the lowest cost. Quad rule based stuck node identification process ensures the precise detection of hole with more accuracy. Energy efficient healing help to decrease the cost of healing. Through the performance evaluation, MHEAL is evaluated, using different criteria like number of movements, total distance traveled \% coverage and showed that it detects and heals the holes despite the number of nodes with less mobility in various situations. The evaluation results demonstrate that MHEAL shows better performance than HEAL and provides a cost-effective, energy efficient and accurate solution for hole detection and healing in hybrid mobile

\section{References}

[1] W.C. Chu et al., "Location Ifree boundary recognition in mobile wireless sensor networks with a distributed approach," Direct Computer Networks, vol. 70, pp. 96-112, 2014..

[2] Z. J. Zhang R. J. S. Fu, , and H. C. Chao,, "energy-efficient motion strategy for mobile sensors in mixed wireless sensor networks," Journal of Distributed Sensor Networks , pp. 12, 2013.

[3] S. Zeadally, N. Jabeur, and I. M. Khan, " Hop-based approach for holes and boundary detection in wireless sensor networks,," in IET Wireless Sensor Systems, vol. 2, no. 4, pp. 328-337, December 2012.

[4] S. R. Mustapha and A. K. Abdelhamid Mellouk, "Localized movement-assisted sensor deployment algorithm for hole detection and healing," IEEE Transactions on Parallel and Distributed Systems, vol. 1, no. 1, 2014. 